\title{
Elimination du CODB par une combinaison de traitements biologiques dont la filtration lente dans une filière de production d'eau potable

Removal of CODB by combination of biological treatments
especially slow sand filtration in drinking water treatment
plan

B. Welté et A. Montiel

Volume 5, numéro hors-série, 1992

URI : https://id.erudit.org/iderudit/705158ar

DOI : https://doi.org/10.7202/705158ar

\section{Aller au sommaire du numéro}

\section{Éditeur(s)}

Université du Québec - INRS-Eau, Terre et Environnement (INRS-ETE)

\section{ISSN}

0992-7158 (imprimé)

1718-8598 (numérique)

\section{Découvrir la revue}

Citer cet article

Welté, B. \& Montiel, A. (1992). Elimination du CODB par une combinaison de traitements biologiques dont la filtration lente dans une filière de production d'eau potable. Revue des sciences de l'eau / Journal of Water Science, 5, 165-176. https://doi.org/10.7202/705158ar

\section{Résumé de l'article}

Dans le cadre de la rénovation de l'usine d'Ivry sur Seine qui assure l'alimentatiop en eau potable de la ville de Paris avec un débit nominal de 300 $000 \mathrm{~m}^{3} / \mathrm{j}$, une nouvelle chaîne originale de traitement a été mise en œuvre. L'originalité de cette chaîne de traitement tient au fait que l'épuration par biodégradation a été prise en compte au niveau de toutes les étapes de traitement. Cette chaîne est divisée en quatre parties : préozonation coagulation sut filtre - filtration biologique lente sur sable et affinage par ozonation filtration sur charbon actif en grains puis désinfection finale au chlore.

Nous avons testé sur pilote l'efficacité de cette nouvelle filière pour l'élimination du carbone organique dissous biodégradable ainsi que l'influence de la préozonation pour l'efficacité de la filtration biologique. Les résultats montrent des performances remarquables de la filtration biologique lente concernant l'élimination du carbone organique biodégradable. L'ozone améliore les performances de la filtration lente, et nous avons observé un taux de traitement optimal correspondant à $0,12 \mathrm{mg} 0_{3} / \mathrm{mg}$ COT. Des corrélations ont été effectuées avec les paramètres globaux de dosage de la matière organique. Elles montrent que le carbone biodégradable issu des filtres biologiques est de nature différente de l'influent et nous estimons qu'il est nécessaire de privilégier une étape d'affinage final par des procédés d'adsorption sur charbon.
Tous droits réservés ( Revue des sciences de l'eau, 1992
Ce document est protégé par la loi sur le droit d'auteur. L’utilisation des services d'Érudit (y compris la reproduction) est assujettie à sa politique d'utilisation que vous pouvez consulter en ligne.

https://apropos.erudit.org/fr/usagers/politique-dutilisation/ 


\title{
Elimination du CODB par une combinaison de traitements biologiques dont la filtration lente dans une filière de production d'eau potable
}

\author{
Removal of CODB by combination \\ of biological treatments especially slow \\ sand filtration in drinking water treatment plan
}

B. WELTÉ1, A. MONTIEL1

Rę̧u le 12 novembre 1991, accepté pour publication le 31 juillet 1992*.

SUMMARY

The city of Paris has decided to improve the Ivry/Seine plant which participates to the water supply of the city. The flow of this plant is $300000 \mathrm{~m}^{3} /$ day and SAGEP has imagined a new line of treatment which takes in account the biodegradation at all the treatment steps. The line of treatment is divided into 4 steps : preozonation, contact coagulation, coagulation on filter, slow sand filtration and ozonation, filtration on GAC, then final disinfection with chlorine. This new line of treatment has been tested on a pilot plant and we have first verified the efficiency of this plant to remove BDOC.

The material used for the contact coagulation is biolite and we have observed a good removal of BDOC on this step. A process of bioflocculation has been shown on this step. Nevertheless, whatever the concentration of BDOC in the raw water, the concentration of BDOC of the effluent water of slow sand filters is atways very low $\left(0,3 \mathrm{mg} . \mathrm{I}^{-1}\right)$. We have measured also others parameters concerning organic matter.

We observed a good removal of UV absorption and fluorescence along the whole water treatment line but the ratio of the fluorescence compared to the dissolved organic carbon increases after slow sand filtration. These observations conlirm what was shown previously. Secondly, we have tested the influence of preozonation on the efficiency of slow sand filtration.

Preozonation has a different efficiency depending on the nature of dissolved organic carbon in the raw water. Preozonation improves efficiency of slow sand filtration but we have observed an optimal ratio of $0,12 \mathrm{mg} 0_{3} / \mathrm{mg}$ TOC. If the dose of ozone is over this ratio, we observed a higher concentration of BDOC in the water flowing out the slow sand fitters.

1. SAGEP Eau de Paris, Mission Qualité, 9 rue Schoelcher, 75014 Paris, France.

* Les commentaires seront rę̧us jusqu'au 30 juin 1993. 
In conclusion, we can say that origin of BDOC is different in the influent and effluent of slow sand filtration and we think that it is necessary to privilege a step of adsorption on GAC after slow sand filtration.

Key-words : treatment, drinking water, biodegradable organic carbon, slow sand fittration

RESUMÉ

Dans le cadre de la rénovation de l'usine d'Ivry sur Seine qui assure l'alimentatio en-eau potable de la ville de Paris avec un débit nominal de $300000 \mathrm{~m}^{3} /$, une nouvelle chaine originale de traitement a été mise en ceuvre. L'originalité de cette chaîne de traitement tient au fait que l'épuration par biodégradation a été prise en compte au niveau de toutes les étapes de traitement. Cette chaîne est divisée en quatre parties : préozenation - coagulation sur filtre - filtration biologigue lente sur sable et affinage par ozonation filtration sur charbon actif en grains puis désinfection finale au chlore.

Nous avons testé sur pilote l'efficacité de cette nouvelle filière pour l'élimination du carbone organique dissous biodégradable ainsi que $I$ influence de la préozonation pour l'efficacité de la filtration biologique. Les résultats montrent des performances remarquables de la filtration biologique lente concernant I'élimination du carbone organique biodégradable. L'ozone améliore les performances de la filtration lente, et nous avons observé un taux de traitement optimal correspondant a $0,12 \mathrm{mg} 0_{3} / \mathrm{mg}$ COT. Des corrélations ont eté effectuées avec les paramétres globaux de dosage de la matière organique. Elles montrent que le carbone biodégradable issu des filtres biologiques est de nature différente de l'influent et nous estimons qu'il est nécessaire de privilégier une étape d'affinage final par des procédés d'adsorption sur charbon.

Mots clés : traitement, eau potable, carbone organique biodégradable, fittration lente.

\section{INTRODUCTION}

Les problèmes de dégradation de l'eau dans les réseaux de distribution sont aujourd'hui d'actualité et il est reconnu qu'on ne peut dissocier l'usine de traitement d'une part et le réseau de distribution d'autre part (HITSCH et WERNER, 1988 ; BLOCK et al., 1989). Dans le cadre de la rénovation de l'usine d'Ivry qui participe pour un tiers à l'alimentation en eau de Paris, la SAGEP a mis au point une filière de traitement nouvelle tout en conservant une étape de filtration biologique lente (MONTIEL et al., 1989), afin de prendre en compte les connaissances les plus récentes sur la dégradation biologique de l'eau dans le réseau de distribution.

Le but de l'étude a été dans un premier temps de vérifier l'efficacité de cette future filière vis-à-vis de l'élimination du carbone organique biodégradable et de mettre en évidence l'influence de la préozonation sur l'efficacité de la filtration biologique à faible vitesse $(5 \mathrm{~m} / \mathrm{jour})$. 


\section{MATÉRIEL ET MÉTHODES}

\section{Etude pilote}

L'étude a été effectuée sur un pilote alimenté en eau de Seine, simulant exactement la future chaine de traitement. Le schéma de ce pilote traitant un débit de $45 \mathrm{I}^{\mathrm{h}} \mathrm{h}^{-1}$ est representé sur la figure 1.

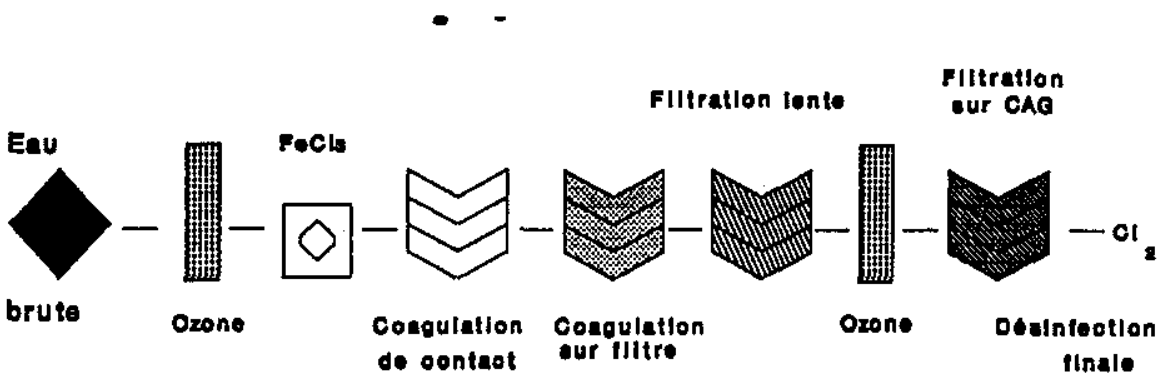

Figure 1 Schéma de la chaîne de traitement pilote.

Scheme of the pilot plant line of treatment.

Les caractéristiques du pilote sont décrites dans le tableau 1.

Tableau 1 Matériaux utilisés dans l'étude pilote.

Table 1 Materials used in pilot plant study.

\begin{tabular}{|lccc|}
\hline \multicolumn{1}{|c}{ Etape } & Matériau utilise & Taille effective & Vitesse de filtration \\
\hline Coagulation de contact & Biolite & $270 \mathrm{~mm}$ & $9 \mathrm{~m} \cdot \mathrm{r}^{-1}$ \\
Coagulation sur filtre & Sable & $0,95 \mathrm{~mm}$ & $6 \mathrm{mh}-1$ \\
Filtration lente & Sable & $0,96 \mathrm{~mm}$ & $5 \mathrm{~m} \cdot-1$ \\
Charbon actif en grains & Charbon & $0,60 \mathrm{~mm}$ & $6 \mathrm{~m} \cdot \mathrm{h}-1$ \\
& CHEMVIRON F400 & & \\
\hline
\end{tabular}

Deux types d'expérimentation ont été faites: dans la première partie de l'étude, le taux d'ozone appliqué en préozonation a été fixé à $1 \mathrm{ppm}$. Le taux d'ozonation appliqué après la filtration lente a été fixé afin d'avoir toujours des conditions virulicides (résiduel de $0,4 \mathrm{ppm}$ au bout de $4 \mathrm{mn}$ de temps de contact). Dans la deuxième partie, nous avons fait varier les doses d'ozone appliquées en préoxydation. Le coagulant utilisé était du chlorure ferrique et le taux de traitement a varié entre 5 et $10 \mathrm{~g} / \mathrm{m}^{3}$. L'alginate de sodium a été utilisé comme adjuvant de floculation à une dose de $0,1 \mathrm{~g} / \mathrm{m}^{3}$. 


\section{Méthodes analytiques}

- La turbidité a été mesurée par nephelométrie. (Norme AFNOR NFT90033)

- La matière organique a été suivie par plusieurs méthodes permettant des approches quantitatives et qualitatives:

- Oxydabilité au permanganate de potassium (norme AFNOR NFT 90018) à chaud en milieu acide (AFNOR, 1986).

- Le carbone organique total (COT) et le carbone organique dissous (COD) ont été determinés à l'aide d'un analyseur de carbone Dohrman DC80, système d'oxydation UV/persulfate et détection du $\mathrm{CO}_{2}$ forme par cellule infrarouge. La précision du dosage a été estimée à $5 \%$.

- Absorption UV à $254 \mathrm{~nm}$.

- Emission de fluorescence à $450 \mathrm{~nm}$ pour deux longueurs d'onde d'excitation $(220 \mathrm{~nm}$ et $260 \mathrm{~nm}$ ). Les valeurs sont exprimées par rapport à l'émission de deux solutions étalon de sulfate de quinine à 100 et $500 \mu \mathrm{g} . \mathrm{I}^{-1}$ (KALLEL, 1988).

- Carbone organique dissous biodégradable (CODB). Au cours de cette étude, nous avons utilisé deux méthodes différentes de détermination du CODB basées sur le suivi de COD :

- La méthode JORET et LEVI (1988) utilisant un inoculum de bactéries fixées autochtones (sable). L'inoculum est constitué par du sable des préfiltres de l'usine d'Ivry (100 g pour $300 \mathrm{ml}$ d'eau) abondamment lavé à l'eau du réseau puis à l'eau distillée jusqu'à absence de relargage de COD détectable. L'incubation a lieu à $20^{\circ} \mathrm{C}$ pendant 7 jours et des prélèvements pour analyse du COD ont été réalisés tous les jours. Nous n'avons pas pratiqué d'aération des échantillons.

- La méthode SERVAIS et BILLEN (1987) utilisant un inoculum de bactéries libres mixtes autochtones (eau brute). Les échantillons ont été ensemencés avec de l'eau de Seine préalablement filtrée sur $2 \mu \mathrm{m}$ (3ml pour $500 \mathrm{ml}$ d'échantillon). L'incubation a lieu à $20^{\circ} \mathrm{C}$ pendant 28 jours et des prélèvements pour analyse du COD ont été réalisés une fois par semaine. Contrairement au protocole proposé par SERVAIS et BILLEN (1987) nous n'avons pas filtré les eaux à étudier sur membrane de porosité $0,2 \mu \mathrm{m}$ avant l'inoculation, ceci afin de prendre en-considération toute la charge organique de l'eau de Seine et de pouvoir établir des comparaisons avec la méthode JORET et LEVI (1988).

\section{RÉSULTATS ET DISCUSSION}

\section{Evolution du CODB le long de la filière}

Pendant toute la durée de l'étude nous avons suivi le comportement de la filière pilote vis-à-vis de l'élimination du carbone organique biodégradable. Les mesures du carbone organique biodégrable ont été effectuées par la méthode JORET et LEVI (1988). Le tauX de préozonation appliqué était toujours de $1 \mathrm{ppm}$. 
Les résultats sont présentés dans la figure 2.

\section{MAI 1989}

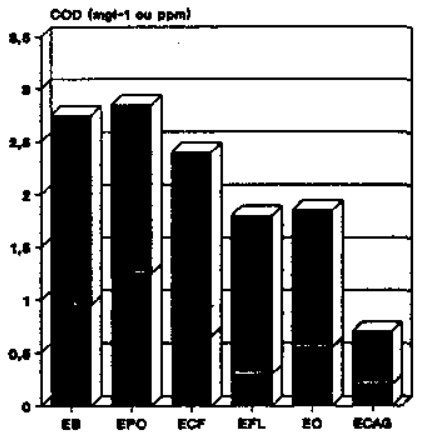

JUILLET 1989

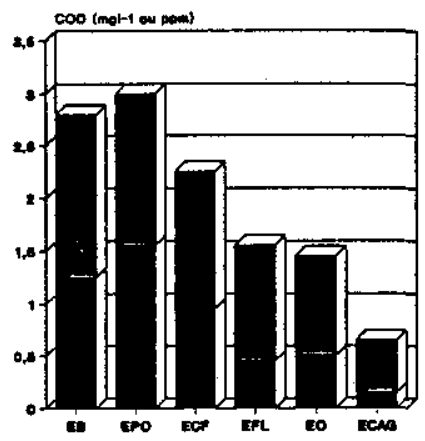

SEPTEMBRE 1989

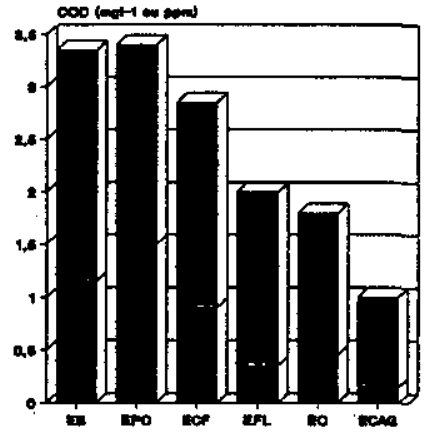

CODB

Figure 2 Evolution du COD et du CODB le long de la filière de traitement à différentes périodes.

Evolution of $C O D$ and $C O D B$ at differents steps of treatment. 


\section{Eau brute}

L'eau de Seine présente des teneurs en carbone dissous variables.

La concentration en carbone organique biodégradable est à peu près constante et représente 30 à $40 \%$ du carbone dissous. La préozonation conduit toujours à une augmentation de la teneur en carbone organique biodégradable.

\section{Coagulation de contact - coagulation sur filtre}

L'étape de coagulation de contact-coagulation sur filtre est très efficace pour l'abattement du carbonē organique biodégradable. En moyenne, sur les trois périodes étudiées, on constate un abattement d'environ $30 \%$ alors que l'abattement total du carbone organique dissous est de 15 à $20 \%$. Les rapports $\mathrm{CODB} / \mathrm{COD}$ diminuent également, ce qui prouve que le CODB est bien éliminé par cette étape.

Les paramètres globaux de la matière organique mesurés (tableaux 2 et 3) montrent un abattement important des molécules responsables de l'absorption UV et de l'émission de fluorescence ( $45 \%$ en moyenne pour l'absorption UV et 50 à $70 \%$ pour la fluorescence).

Tableau 2 Evolution de l'absorption UV et de la fluorescence à toutes les étapes de la filière.

Table 2 Evolution of UV absorption and fluorescence at all steps of pilot plant.

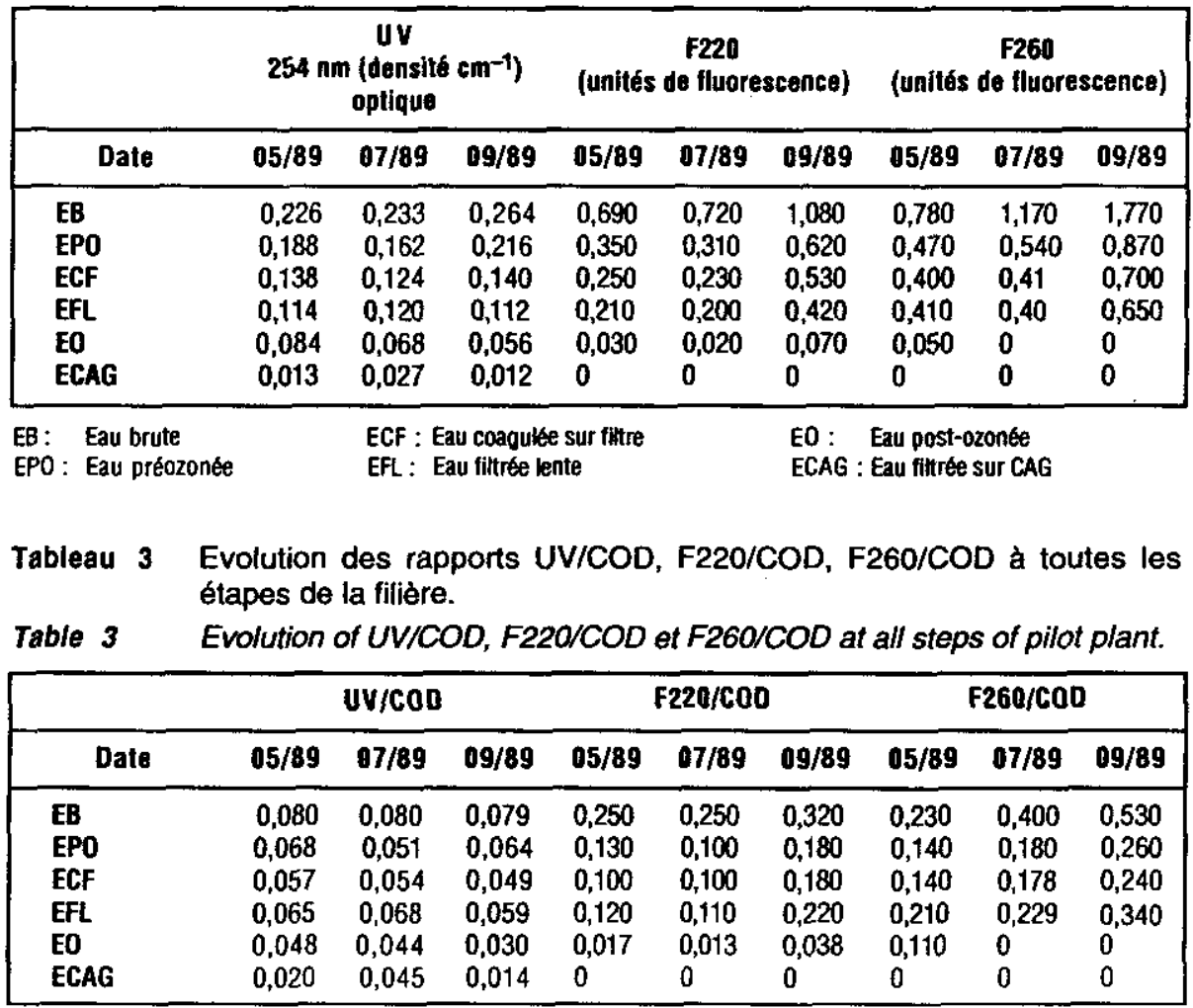


Si on prend en compte la fraction de matière organique caractérisée par les molécules absorbant I'UV ou fluoresçant, on voit que ce pourcentage diminue par l'étape de coagulation de contact-coagulation sur filtre (tableau 3) (en moyenne, $8 \%$ dans l'eau brute et 5 à $6 \%$ dans l'eau après coagulation sur filtre pour l'absorption UV et $25 \%$ pour la fluorescence dans l'eau brute $(\lambda=220 \mathrm{~nm})$ contre 10 à $18 \%$ dans l'eau après coagulation sur fiftre). Ceci montre que dans cette étape, la fraction de CODB éliminée correspond à des molécules qui possèdent des chromophores (absorption UV et émission de fluorescence). Ces molécules ont été déjà bien éliminées par préozonation. Or, au niveau de cette étape, nous ne pratiquons pas une coagulation au taux optimal de traitement (le 1/10 de la dose obtenue avec essais Jar test est mis en œuvre dans ce prétraitement). L'abattement important de carbone organique biodégradable peut se faire par 2 mécanismes :

- coagulation de la matière organique

- processus de biofloculation

Les résultats nous ont montré un abattement important du CODB, l'abattement du COD étant faible, ceci est logique si on considère que cet abattement est du uniquement au processus de coagulation, la dose de coagulant utilisé étant très faible.

Nous avons voulu mettre en évidence le processus de biofloculation. Pour cela, nous avons comparé 2 matériaux: la biolite que nous utilisons dans l'étape de coagulation de contact et du gravier de même granulométrie.

Les résultats présentés sur les figures 3 et 4 montrent un abattement de turbidité bien meilleur sur biolite que sur gravier ainsi qu'une nitrification très importante. La biolite est un matériau où une activité biologique intense se développe, ce qui permet des phénomènes de biofloculation et donc un bon abattement du CODB.

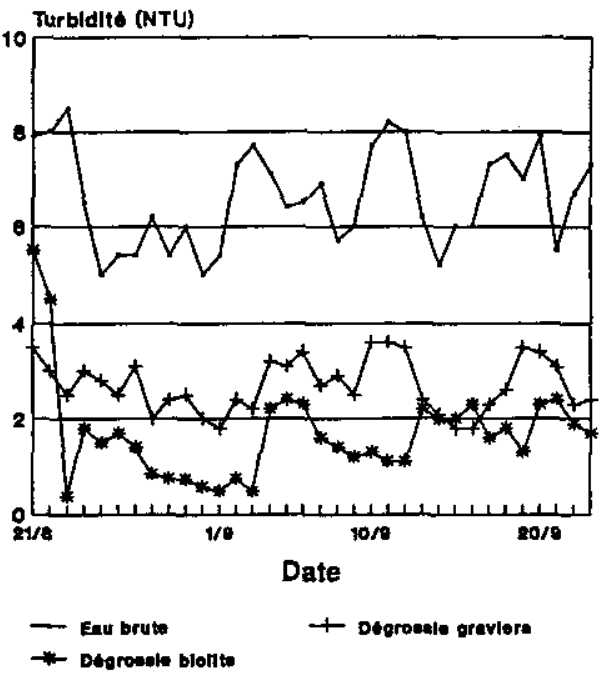

Figure 3 Mise en évidence de la biofloculation sur la biolite.

Evidence of bioflocculation on biolite. 


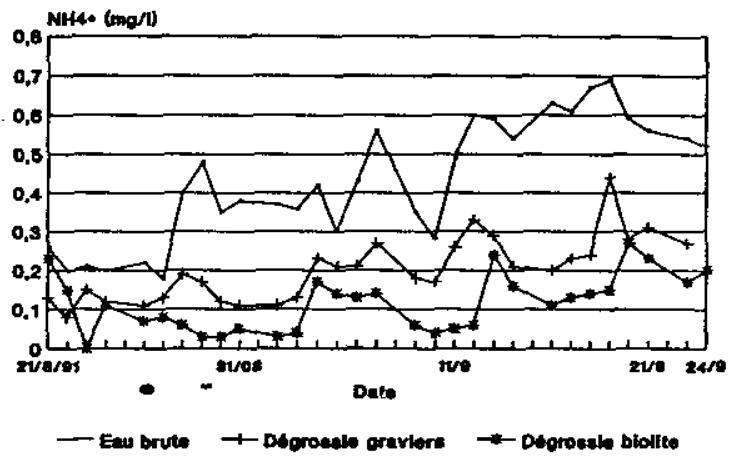

Essais du2V08/91 au 30/09/91

Figure 4 Mise en évidence de la nitrification biologique sur biolite.

Evidence of biologic denitrification on biolite.

\section{Flitration lente}

Les résultats de la figure 2 montrent un très bon abattement du CODB par filtration lente $(50$ à $60 \%$ ) et un abattement du COD relativement important (27 à $35 \%$ ). Malgré tout, le rapport CODB/COD diminue encore (il passe en moyenne de $35 \%$ après coagulation sur filtre à $20 \%$ après filtration lente). Les teneurs en CODB dans l'eau issue des filtres lents sont toujours très faibles.

La filtration lente permet également un abattement de 20 à $30 \%$ du carbone réfractaire. Les mécanismes mis en jeu lors de cette étape de filtration biologique sont de 2 ordres :

- élimination par biodégradation

- élimination par adsorption (MONTIEL et WELTE, 1991).

Si on étudie les paramètres globaux de caractérisation de la matière organique (tableau 3), on note une augmentation par rapport à l'eau coagulée sur filtre des valeurs de fluorescence ou d'absorption UV rapportées au carbone organique dissous après la filtration lente.

Ce phénomène pourrait être dû à une moins bonne élimination des composés responsables de l'absorption UV et de l'émission de fluorescence tels que les substances humiques. VAN DER KOOIJ (1987) a montré qu'il existait une relation linéaire entre l'abattement d'absorption UV et l'augmentation de carbone organique assimilable. Nous avons vérifié ceci et nous avons déjà montré que l'on pouvait faire une corrélation entre ces paramètres ef le carbone réfractaire, (augmentation du rapport UV/COD avec augmentation du carbone réfractaire) ces composés étant plus particulièrement à l'origine de l'émission de fluorescence et de l'absorption UV (BONNET et al., 1991).

Nous pouvons dire que les eaux après filtration lente ont une grande stabilité biologique vu l'augmentation des rapports F220/COD et UVCOD. L'hypothèse de cette augmentation est la production par les micro-organismes des filtres lents, de sous produits de leur métabolisme, composés essentiellement réfractaires. 


\section{Couplage ozone charbon actif}

L'ozonation pratiquée après la filtration lente ne provoque pas toujours une augmentation du carbone organique biodégradable: ceci confirme que les molécules biodégradables et facilement oxydables par l'ozone ont été éliminées lors de la filtration lente.

Par contre, on note un abattement très important des molécules responsables de la fluorescence (85 à $90 \%$ ).

Enfin, nous pouvons dire que l'étape de filtration sur charbon actif en grains ayant des propriétés adsorbantes importantes (le charbon actif était neuf lors de cette étude) permet d'obtenir des teneurs en CODB très basses et pout conduire à l'obtention d'une eau ayant une grande stabilité biologique dans le réseau de distribution. L'abattement optimal de l'absorption UV est également obtenu lors de cette étape.

\section{Influence de la préozonation}

Plusieurs taux d'ozone ont été testés en préoxydation et nous avons comparé l'efficacité de la filtration lente en fonction des taux d'ozone appliqués en amont.

L'étude a été poursuivie sur le même pilote mais en ne prenant en compte que les étapes jusqu'a la filtration lente. Le suivi du carbone organique biodégradable a été effectue par la méthode de Servais et Billen. Deux périodes ont été étudiées:

- au mois d'avril

- au mois de mai

Les caractéristiques de la matière organique pour ces deux périodes sont données dans le tableau 4. On voit que les teneurs sont différentes mais il faut noter que la nature de la matière organique est également différente : au mois de mai, nous avons observé une très forte crue plantonique alors qu'au mois d'avril, les teneurs en matière organique sont comparables à celles observées pendant l'hiver et dont l'origine est vraisemblablement la matière humique.

Tableau 4 Caractéristiques de la matière organique dans l'eau au sein des 2 périodes étudiées.

Table 4 Characteristics of organic matter in water during the 2 periods studied.

\begin{tabular}{|c|c|c|c|c|c|}
\hline & $\cot m g /$ & $\begin{array}{c}\text { Oxydabilité } \\
\mathrm{mg} / / \mathrm{O}_{2}\end{array}$ & $\begin{array}{c}\text { UV } \\
\text { (densite optlque) }\end{array}$ & $\begin{array}{l}220 \mathrm{~nm} \text { (unith de } \\
\text { fluorescence) }\end{array}$ & $\begin{array}{l}260 \mathrm{~nm} \text { (unit6 de } \\
\text { fluorescence) }\end{array}$ \\
\hline Avril & 4,4 à 5,1 & 6,5 a 7,5 & 0,20 & 0,85 & 0,99 \\
\hline $\mathrm{Ma}$ & 3 a 4 & 5 a 6,8 & 0,29 & 1,50 & 2,20 \\
\hline
\end{tabular}

Pour des teneurs en carbone organique dissous comparables dans l'eau brute, les proportions relatives de carbone biodégradable sont très différentes (fig. 5) (11\% en moyenne en avril contre $42 \%$ en mai) (fig. 4). Cela confirme I'hypothèse de la "nature différente" de la matière organique. 

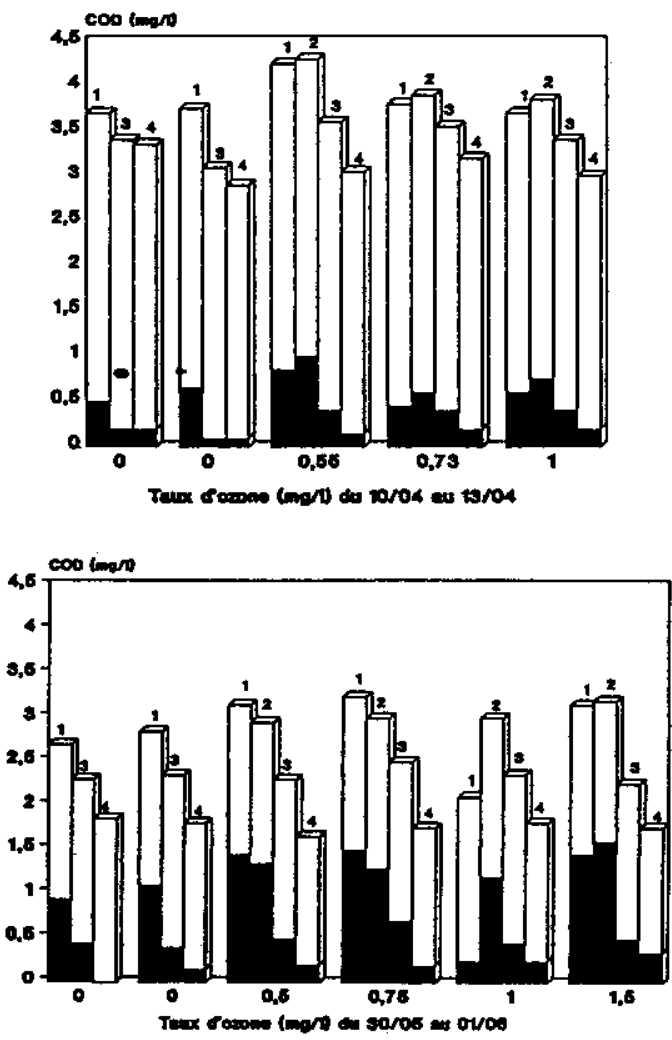

CODR

COOB

( 1 : eau brute ; 2 : eau précozonse ; 3 : eau apres coagulationffiltration ; 4 : eau apres filtration lente)

Figure 5 Evolution des valeurs de COD, CODB et CODR pour différents taux de préozonation et pour les deux périodes d'essais.

Influence of dose of $\mathrm{O}_{3}$ on the evolution of $D O C$ (BDOC and $\mathrm{RDOC}$ ).

L'ozone a une action très différente sur le carbone organique dissous : au mois d'avril, nous observons une augmentation du carbone biodégradable qui a été souvent observée. Par contre, au mois de mai nous avons une diminution du carbone organique biodégradable après l'étape de préozonation. Ceci est simplement dû à la composition différente de la matière organique et en présence d'algues, nous avons un phénomène de microfloculation. Ce phénomène a souvent été cité (RECKHOW, 1986 ; MAIER, 1974) avec libération de biopolymères par lyse de cellules algales, permettant une meilleure coagulation de la matière organique. Après préozonation, nous avons observé une augmentation du CODB et ce au détriment de la fraction réfractaire, puis qu'aucune modification significative du COD n'est apparue. Cependant, en mai, nous n'avons observé qu'üne faible augmentation des teneurs en CODB. L'élimination du carbone organique biodégradable se fait essentiellement au 
niveau de l'étape de coagulation de contact. L'ozonation joue un rôle différent dans les deux cas : au mois d'avril, la préozonation défavorise l'élimination du CODB par coagulation de contact mais améliore l'élimination par filtration lente (passage de $60 \%$ d'élimination sans préozonation à $25 \%$ avec, pour la coagulation de contact et de $0 \%$ sans préozonation contre $50 \%$ avec, pour la filtration lente). Au mois de mai au contraire, nous avons un très bon abattement du CODB par coagulation de contact. Les pourcentages d'abattement avec l'ozone sur la coagulation de contact/coagulation sur filtre atteignent $65 \%$ et par contre, les pourcentages d'abattement sur la filtration lente passent de $80 \%$ sansopréozonation à $30 \%$ avec la dose maximale d'ozone appliquée.

Au total, nous avons observé une élimination maximale de $28 \%$ du COD en avril pour un taux d'ozone de $0,11 \mathrm{mg} 0_{3} / \mathrm{mg}$ COT et de $48 \%$ en mai pour un taux d'ozone de $0,14 \mathrm{mg} 0_{3} / \mathrm{mg}$ COT. Dans tous les cas, nous avons une teneur très faible en carbone organique biodégradable issu des filtres lents. Par contre, pour des taux d'ozone élevés, on constate une augmentation du carbone organique biodégradable issu des filtres lents (passage de 0,35 $\mathrm{mg} / \mathrm{l}$ sans ozone à $0,50 \mathrm{mg} / \mathrm{l}$ pour un taux de traitement de $0,43 \mathrm{mg} 0_{3} / \mathrm{mg}$ COT). Nous confirmons ce que nous avons dit dans la première partie de l'étude : le carbone biodégradable issu des filtres lents est de nature différente et l'activité biologique sur les filtres lents est augmentée après la préozonation, produisant des sous produits en quantité plus importante.

\section{CONCLUSION}

L'expérimentation pilote menée sur plus d'un an nous a montré que la chaîne de traitement prévue à l'usine d'Ivry était très performante pour l'élimination du carbone organique biodégradable. Les étapes de filtration biologique lente et de filtration sur charbon actif en grains permettent d'avoir des eaux ayant des teneurs en carbone biodégradable très faibles, stables biologiquement. Néanmoins, malgré un abattement également important du carbone réfractaire, il peut rester des molécules sensibles encore à l'ozone. D'autre part, la vie biologique à l'intérieur des filtres biologiques étant très intense, nous pouvons avoir relargage de sous-produits ce qui peut se traduire par une augmentation relative du carbone organique biodégradable. En conséquence, il est important d'utiliser une étape de filtration sur charbon actif en grains suivant la filtration lente favorisant les processus d’adsorption.

La préozonation favorise en général l'élimination de la matière organique le long de la filière mais surtout en période algale quand la teneur en carbone organique biodégradable est déjà très élevée dans l'eau brute. 


\section{RÉFÉRENCES BIBLIOGRAPHIQUES}

AFNOR Eau 1986 Méthodes d'essai. Recueil des normes françaises.

BLOCK J.C., PAQUIN J.L., COLIN F., LEVI Y., 1989. Poster présentation: les développernents biologiques en réseau. Un nouvel outil pour de nouvelles approches théoriques et expérimentales. Water Supply: 7.

BONNET M.C., WELTE B., MONTIEL A., DORE M., 1991. Elimination du carbone organique biodégradable par les procédés de traitement biologique sur les filières de production d'eau potable. Environmental Technology, 12, 217-229.

JORET J.C., LEVY Y., DUPIN T., GIBERT M., 1988. Rapid method for estimating bioeliminable organic carbon in water. In "The wonderful world of water". AWWA, Annual Conference Proceedings, Orlando 19-23 juin : 1715-1725.

KALLEL S., 1988. DEA hydrologie : Mesure de la fluorescence : application au contrôle en continu de la qualité de l'eau. Université Pierre et Marie Curie.

MAIER D., 1978. Microfloculation par l'ozone. Procédés d'oxydation appliqués au traitement de l'eau potable. Proceedings Kartsruhe : 435-462

MONTIEL A., WELTE B., 1991. Altemative options for atrazine on water treatment device - presented at 1st IAWPRC Symposium on hazard assessment and control of environmental contaminants in the water OTSU City 25-28 November.

MONTIEL A., WELTE B., BARBIER J.M., 1989. Improvement of slow sand filtration. Environmental Technology Letters, 10 : 29-40.

RECKHOW D.A., SINGER P.C., TRUSSEL R.R., 1986. Ozone as coagulant aid presented at the 1986 AWWA National Conference, June: 1-8 (In AWWA Seminar Proc : ozonation recent advances and research needs. AWWA, DENVER, $\mathrm{CO}$ ).

SERVAIS P., BILLEN G., HASCOET M.C., 1987. Determination of the biodegradable fraction of dissolved organic matter in water. Water Research, 21 : 445-490.

VAN DER KOOIJ D., HIJNEN W.A.M., KRUITHOF J.C., 1987. The effects of ozonation, biological filtration and distribution on the concentration of easily assimilable organic carbon (AOC) in drinking water. In Proceedings of 8 th ozone world congress, Zurich, 15-18 sept., 1 : D96-D113.

WERNER P., HAMBSCH B., 1988. Messung der Wachstumrate von bakterien bei der aufbereitung von oberflachenwassern. Vom wasser, 70 : 233-244. 